\title{
Vacancy-type defects in Er-doped GaN studied by a monoenergetic positron beam
}

\author{
A. Uedono, ${ }^{1, a)}$ C. Shaogiang, ${ }^{1}$ S. Jongwon, ${ }^{1}$ K. Ito, ${ }^{1}{ }^{H}$. Nakamori, ${ }^{1}$ N. Honda, ${ }^{1}$ \\ S. Tomita, ${ }^{1}$ K. Akimoto, ${ }^{1}$ H. Kudo, ${ }^{1}$ and S. Ishibashi ${ }^{2}$ \\ ${ }_{1}^{1}$ Institute of Applied Physics, University of Tsukuba, Tsukuba, Ibaraki, 305-8573, Japan \\ ${ }^{2}$ Research Institute for Computational Sciences, National Institute of Advanced Industrial Science \\ and Technology, Tsukuba, Ibaraki, 305-8568, Japan
}

(Received 20 January 2008; accepted 20 March 2008; published online 22 May 2008)

\begin{abstract}
A relationship between intra- $4 f$ transitions of Er and vacancy-type defects in Er-doped GaN was studied by using a monoenergetic positron beam. Doppler broadening spectra of the annihilation radiation were measured for Er-doped GaN grown by molecular beam epitaxy. A clear correlation between the defect concentration and the photoluminescence (PL) intensity was observed. The major defect species detected by positrons was identified as a Ga vacancy $V_{\mathrm{Ga}}$, and its concentration increased with increasing Er concentration $[\mathrm{Er}]$. For the sample with $[\mathrm{Er}]=3.3$ at. \%, the maximum integrated intensity of PL was observed. The $V_{\mathrm{Ga}}$ concentration was above $10^{18} \mathrm{~cm}^{-3}$ and additional vacancies such as divacancies started to be introduced at this Er concentration. For the sample with higher [Er], the PL intensity decreased, and the mean size of vacancies decreased due to an introduction of precipitates and/or metastable phases. (C) 2008 American Institute of Physics.
\end{abstract}

[DOI: $10.1063 / 1.2932166$ ]

\section{INTRODUCTION}

Optical properties of semiconductors doped with rareearth $(\mathrm{RE})$ metals have been extensively studied due to their potential in device applications such as full color displays and telecommunication ${ }^{1}$ equipment. Photoluminescence (PL) from intra- $4 f$ shell transitions from RE metals shows sharp emission lines which match red-green-blue primary colors, and their wavelengths are stable with host materials and temperature variations. Among the many possible host materials, wide gap semiconductors, such as $\mathrm{GaN}$, are advantageous because of the emissions of high-energy RE transitions adsorbed in narrow band-gap semiconductors. Erbium (Er) has been an intensively investigated RE metal because it exhibits emission lines in infrared (IR) $(1.54 \mu \mathrm{m})$ as well as in green emission (537 and $558 \mathrm{~nm}$ ); the IR region can be used to reduce loss in silica fibers used for telecommunications. ${ }^{2-7}$ In the trivalent charge state of Er, $4 f$ electrons are shielded from the host by filled $5 s$ and $5 p$ shells. The transition between the first excited state $\left({ }^{4} I_{13 / 2}\right)$ and the ground state $\left({ }^{4} I_{15 / 2}\right)$ gives rise to the IR emission and the green emission lines are produced by the transition between ${ }^{2} H_{12 / 2}$ or ${ }^{4} S_{3 / 2}$ and the ground state.

Erbium doping into GaN has been done through several techniques such as ion implantation, ${ }^{2,4}$ metal-organic chemical vapor deposition, 3,5 and molecular beam epitaxy (MBE). ${ }^{3,7}$ The emission efficiency of Er-doped GaN, however, depends on the growth conditions of the GaN, and devices based on GaN suffer from low quantum efficiency. It was reported that the intraemission from a RE metal correlated with vacancies adjacent to $\mathrm{Er}^{5-7}$ It was also reported that PL intensities are strongly influenced by the presence of a special distribution of point defects and dislocations. ${ }^{8}$

${ }^{a)}$ Electronic mail: uedono@sakura.cc.tsukuba.ac.jp.
Thus, a fundamental understanding of the behavior of point defects is important to effectively utilize optical devices based on RE-doped GaN. Positron annihilation is a powerful technique for evaluating vacancy-type defects in semiconductors. ${ }^{9}$ Vacancy-type defects in $\mathrm{GaN}$ have been investigated using this method, ${ }^{10-16}$ and the results show that positrons are a powerful probe for studying vacancies and vacancy-impurity complexes in $\mathrm{GaN}$. In the present study, we used a monoenergetic positron beam to probe vacancytype defects in Er-doped GaN grown using MBE.

When a positron is implanted into condensed matter, it annihilates with an electron and emits two $511 \mathrm{keV} \gamma$ quanta. ${ }^{9}$ The energy distribution of the annihilation $\gamma$ rays is broadened by the momentum component of the annihilating electron-positron pair $p_{L}$, which is parallel to the direction of the $\gamma$ rays. The energy of the $\gamma$ rays is given by $E_{\gamma}$ $=511 \pm \Delta E_{\gamma} \mathrm{keV}$. Here, the Doppler shift $\Delta E_{\gamma}$ is given by $\Delta E_{\gamma}=p_{L} c / 2$, where $c$ is the speed of light. A freely diffusing positron may be localized in a vacancy-type defect due to Coulomb repulsion from ion cores. Because the momentum distribution of the electrons in such defects differs from that of electrons in the bulk material, these defects can be detected by measuring the Doppler broadening spectra of the annihilation radiation. The resultant changes in the spectra are characterized by the $S$ parameter, which mainly reflects changes due to the annihilation of positron-electron pairs with a low-momentum distribution, and by the $W$ parameter, which mainly characterizes changes due to the annihilation of pairs with a high-momentum distribution. In general, the characteristic value of $S(W)$ for the annihilation of positrons trapped by vacancy-type defects is larger (smaller) than that for positrons annihilated from the free state. 


\section{EXPERIMENT}

The samples used in the present experiments were 0.8 - $\mu$ m-thick Er-doped GaN grown on (0001) sapphire substrates by gas-source $\mathrm{MBE}$ using $\mathrm{NH}_{3}$ as the nitrogen source. Details of the growth method are given elsewhere. ${ }^{17}$ Metallic $\mathrm{Ga}$ and Er were evaporated from conventional Knudsen effusion cells. Before growth, the substrate was nitridated under an $\mathrm{NH}_{3}$ pressure of $5 \times 10^{-3} \mathrm{~Pa}$ for $10 \mathrm{~min}$ at $940{ }^{\circ} \mathrm{C}$. The substrate temperature was kept at $700{ }^{\circ} \mathrm{C}$ during the growth. No buffer or intermediate layers were formed before the growth of Er-doped GaN. A 3.5- $\mu$ m-thick undoped GaN was also fabricated using growth conditions similar to those described above. PL measurements were done using an $\mathrm{Ar}$ gon laser $(488 \mathrm{~nm})$ at $77 \mathrm{~K}$. The structural properties of the films were evaluated by $\mathrm{X}$-ray diffraction (XRD) using $\mathrm{Cu} K \alpha_{1}$ and $K \alpha_{2}$ radiation. The Er concentrations [Er] and their depth distributions were measured by Rutherford backscattering (RBS) using $30 \mathrm{MeV} \mathrm{O}^{5+}$ obtained from a $12 \mathrm{MV}$ tandem accelerator.

With a monoenergetic positron beam, the Doppler broadening spectra of the annihilation radiation were measured with a Ge detector as a function of the incident positron energy $E$. For each incident positron energy $E$, a spectrum with about $1 \times 10^{6}$ counts was obtained. The low-momentum part was characterized by the $S$ parameter, defined as the number of annihilation events over the energy range of $511 \mathrm{keV} \pm \Delta E_{\gamma}$ (where $\Delta E_{\gamma}=0.76 \mathrm{keV}$ ) around the center of the peak. The high-momentum part of each Doppler broadening spectrum was characterized by the $W$ parameter, which is calculated from the tail of the peak, in the range of $3.4 \mathrm{keV} \leqslant\left|\Delta E_{\gamma}\right| \leqslant 6.8 \mathrm{keV}$. The relationship between $S$ and $E$ was analyzed by VEPFIT, a computer program developed by van Veen et al. ${ }^{18}$ The $S$ - $E$ curve was fitted using

$$
S(E)=S_{s} F_{s}(E)+\Sigma S_{i} F_{i}(E),
$$

where $F_{s}(E)$ is the fraction of positrons annihilated at the surface and $F_{i}(E)$ is the fraction annihilated in the $i$ th layer $\left[F_{s}(E)+\Sigma F_{i}(E)=1\right] . S_{s}$ and $S_{i}$ are $S$ parameters corresponding to the annihilation of positrons on the surface and in the $i$ th layer respectively. To examine the annihilation characteristics of positrons in the damaged region in detail, we used a coincidence-detection system to obtain the Doppler broadening spectra.

Doppler broadening spectra corresponding to the annihilation of positrons in vacancy-type defects in $\mathrm{GaN}$ were theoretically calculated from valence-electron wave functions obtained by the projector augmented-wave (PAW) method $^{19}$ using our in-house QMAS (Quantum Materials Simulator) code. ${ }^{20}$ In this method, since the wave functions exhibit correct behavior near ion cores, the corresponding electron-positron momentum densities in a higher momentum region can be obtained with better accuracy than by using the norm-conserving pseudopotential (NCPP) method. The difference between results obtained by the PAW and NCPP methods is described elsewhere. ${ }^{21}$ The Doppler broadening spectra of positrons trapped by vacancies were calculated in a cell containing about 128 atoms, with the atomic positions fully relaxed by means of $a b$ initio quenched mo-

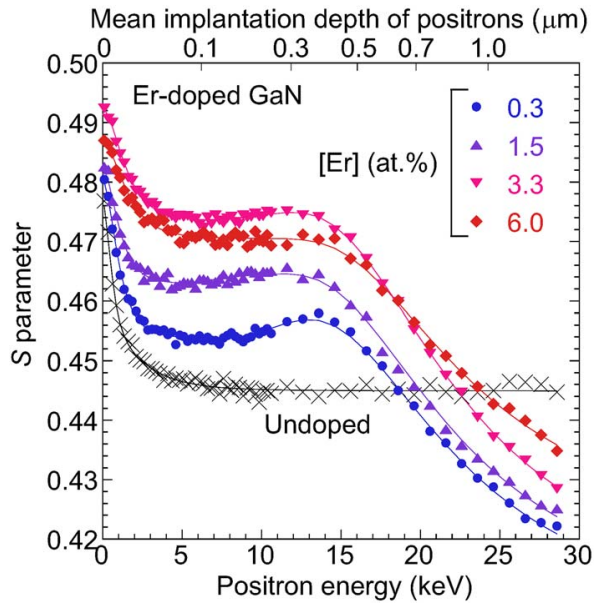

FIG. 1. (Color online) $S$ as a function of incident positron energy $E$ for Er-doped $\mathrm{GaN}$ with $[\mathrm{Er}]=0.3-6.0$ at. \% . The result for undoped $\mathrm{GaN}$ is also shown. The $S$ values at $E \leqslant 14 \mathrm{keV}$ correspond to the annihilation of positrons in the GaN layers. The solid curves are fits to the experimental data. The derived depth distributions of $S$ are shown in Fig. 3.

lecular dynamics. The spectra corresponding to the annihilation of positrons from the free state in $\mathrm{GaN}$ (bulk GaN) were also calculated in a Wigner-Seitz cell containing two atoms as well as a 64-atom cell for comparison. The formalism of the local density approximation ${ }^{22}$ was used in this calculation of the positron wave functions and that of generalized gradient approximation ${ }^{23}$ was used for the electron wave functions.

\section{RESULTS AND DISCUSSION}

The $S$ values of Er-doped GaN with [Er] $=0.3-6.0$ at. \% as functions of $E$ are shown in Fig. 1. Results for undoped GaN are also shown. For the undoped sample, the $S-E$ curve was fitted using Eq. (1). The solid curves are fits to the experimental data. The obtained diffusion length of positrons $L_{d}$ was $31 \pm 3 \mathrm{~nm}$. This value was shorter than that $(51 \mathrm{~nm})$ previously reported for selfstanding GaN fabricated by a lateral epitaxial overgrowth (LEO) technique. ${ }^{24}$ The derived characteristic $S$ value $(0.4449 \pm 0.0002)$ was larger than that for LEO-GaN $(0.44)$. These values indicate that positrons implanted into the undoped GaN film were annihilated from the trapped state by vacancy-type defects. The defect species and its concentration are discussed later.

For the Er-doped samples, the $S$ values at $E \leqslant 14 \mathrm{keV}$ correspond to the annihilation of positrons in the GaN film, and the decrease in the $S$ value above $15 \mathrm{keV}$ was due to the annihilation of positrons in the sapphire substrate. Figure 2 shows the relationship between the $S$ values corresponding to the annihilation of positrons in the GaN film and the $\mathrm{Er}$ concentration. The variation of the integrated intensity of the $511 \mathrm{~nm}$ PL line is also shown. The behavior of the $S$ value was similar to that of the PL intensity, suggesting the vacancy concentration and/or the vacancy species play a crucial role in the intraemission process of Er. The IR emission rate was measured using the cathode-luminescence technique, and emission was observed only for the sample with [Er] $=3.3$ at. \% . The $S$ value increased with increasing Er concen- 


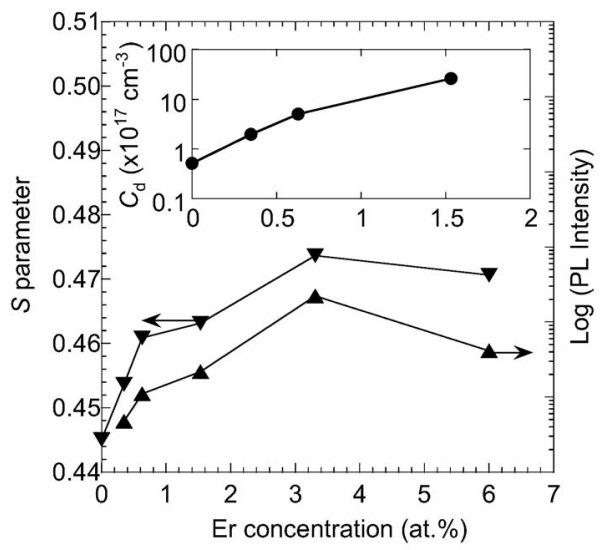

FIG. 2. $S$ values corresponding to the annihilation of positrons in Er-doped $\mathrm{GaN}$ and the integrated intensities of a $551 \mathrm{~nm}$ PL line as a function of the Er concentration, where the PL intensity is shown in a logarithmic scale. The inset shows the concentration of Ga vacancies $\left(V_{\mathrm{Ga}}\right)$ derived from the positron trapping model.

tration $([\mathrm{Er}] \leqslant 3.3$ at. \%), suggesting the introduction of vacancy-type defects due to Er incorporation. Erbium can occupy different lattice locations or form complexes with impurities in $\mathrm{GaN},{ }^{25,26}$ but a Ga lattice site is expected to be the major substitutional site. ${ }^{6}$ The difference between the bond lengths of $\mathrm{Ga}-\mathrm{N}$ and $\mathrm{Er}-\mathrm{N}$ is one cause of the introduction of vacancy-type defects. In GaN, since the charge state of $\mathrm{N}$ vacancies $\left(V_{\mathrm{N}}\right)$ is positive, ${ }^{27}$ the major defect species detected by positrons is $\mathrm{Ga}$ vacancies $\left(V_{\mathrm{Ga}}\right)$ and/or their complexes with impurities. ${ }^{9-16}$ Thus, from Fig. 2, one can conclude that such $V_{\mathrm{Ga}}$-related defects are introduced with increasing Er concentration at $[\mathrm{Er}] \leqslant 3.3$ at. \%. For Er-doped $\mathrm{GaN}$ with $[\mathrm{Er}]=6.0$ at. \%, however, the $S$ value was smaller than that for the sample with $[\mathrm{Er}]=3.3$ at. \%. XRD measurements showed that the (0002) reflection of hexagonal GaN decreased with increasing [Er], and the intensity for the sample with $[\mathrm{Er}]=6.0$ at. \% was two orders smaller than that for $\mathrm{GaN}$ with $[\mathrm{Er}]=0.3$ at. \%. The observed degradation the crystallinity can be attributed to the introduction of precipitates and/or metastable ErN. ${ }^{6,13}$ Thus, the decrease in the $S$ value for this sample can be attributed to the shrinkage of the mean open space of vacancies due to the decrease in the concentration of Er incorporated in the GaN matrix and/or the annihilation of positrons in precipitates or metastable phases.

The $S$ - $E$ relationships for Er-doped GaN were fitted using Eq. (1), and the derived depth distributions of $S$ are shown in Fig. 3. For the sample with $[\mathrm{Er}]=0.3$ at. \%, the $S$ value near the GaN/substrate interface was larger than that in the subsurface region $(<400 \mathrm{~nm})$, suggesting the introduction of vacancy-type defects near the interface. No increase in the $S$ value near the interface was observed for the sample with $[\mathrm{Er}]=0.6$ at. \%, which can be attributed to the increase in the $S$ value near the surface and a resultant masking of the $S$ value near the interface. For the samples with $[\mathrm{Er}]=1.5$ and 3.3 at. \%, similar behavior of $S$ was observed again. A similar enhanced introduction of vacancy-type defects was observed for Eu-doped GaN. ${ }^{13}$ Figure 4 shows the depth distribution of Er measured by RBS for the sample with [Er] $=0.6,1.5$, and 3.3 at. $\%$, where the data were smoothed using

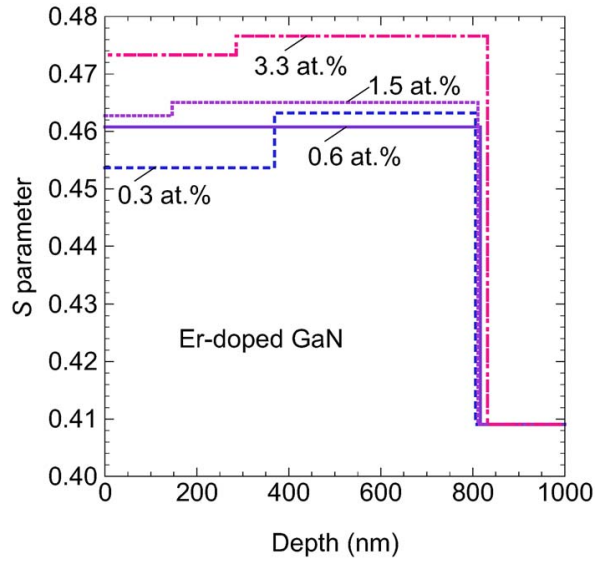

FIG. 3. (Color online) Depth distributions of $S$ for Er-doped GaN with Er concentrations of $0.3-3.3$ at. \% obtained from the analysis of the $S-E$ curves.

FFT technique. The Er concentration near the GaN/substrate interface $(\approx 17.3 \mathrm{MeV})$ was high, suggesting a pile up of $\mathrm{Er}$ at the interface. The large lattice mismatch between $\mathrm{GaN}$ and the substrate makes the interface an appropriate region for reducing the stress caused by the difference in bond lengths of $\mathrm{Er}-\mathrm{N}$ and $\mathrm{Ga}-\mathrm{N}$. Thus, Er is likely to accumulate near the interface and would result in the introduction of vacancytype defects. For the sample with $[\mathrm{Er}]=1.5$ at. \%, accumulation of Er near the subsurface region $(\approx 20.8 \mathrm{MeV})$ was also observed, but this was not the case for other samples. This phenomenon could relate to the change in the potential energy of Er in the GaN matrix with an increasing defect concentration in the film.

Figure 5 shows $(S, W)$ values corresponding to the annihilation of positrons in the damaged region of Er-doped $\mathrm{GaN}$ which were measured by a coincidence-detection system. Results for a GaN substrate grown by hydride vapor phase epitaxy (HVPE) are also shown. ${ }^{28}$ The lifetime spectrum of positrons obtained for this sample was $153 \pm 1$ ps. The derived lifetime is close to the lifetime of positrons that annihilate from the delocalized state, suggesting that the $(S, W)$ value for this sample is close to the characteristic value for

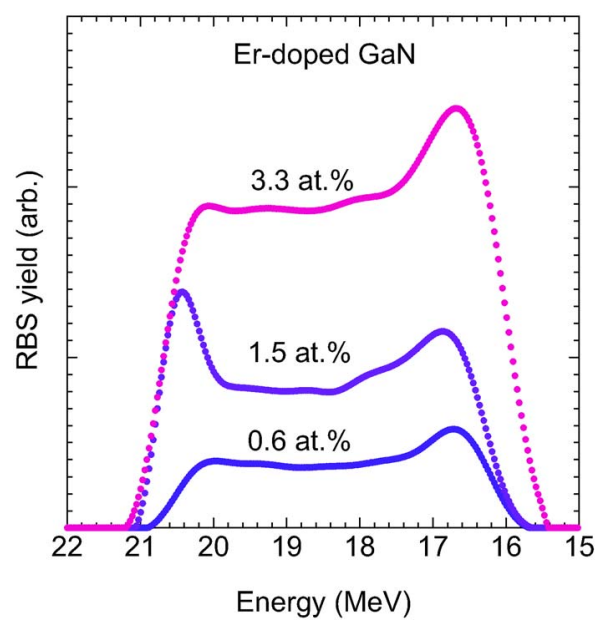

FIG. 4. (Color online) RBS random spectra corresponding to the depth distributions of Er in $\mathrm{GaN}$ with Er concentrations of 0.6, 1.5, and 3.3 at. \%. 


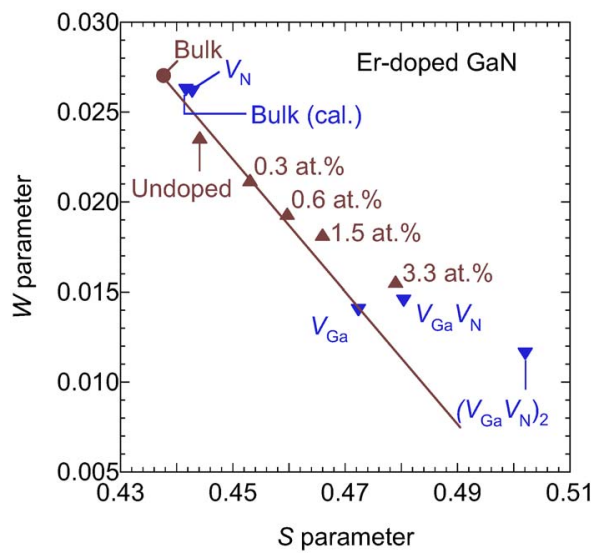

FIG. 5. (Color online) $S$ - $W$ relationships for the annihilation of positrons in undoped and Er-doped GaN grown using $\operatorname{MBE}(\boldsymbol{\Lambda})$. The result for HVPE$\mathrm{GaN}$ was marked as "bulk" $(\boldsymbol{\bullet})$. Values $(\boldsymbol{\nabla})$ obtained through the firstprinciple calculations for the positron annihilation in bulk, Ga vacancy $\left(V_{\mathrm{Ga}}\right)$, N vacancy $\left(V_{\mathrm{N}}\right)$, divacancy $\left(V_{\mathrm{Ga}} V_{\mathrm{N}}\right)$, and quadravacancy $\left[\left(V_{\mathrm{Ga}} V_{\mathrm{N}}\right)_{2}\right]$ are also shown.

defect-free $\mathrm{GaN}$. The $(S, W)$ values obtained through the first-principle calculations are also shown, where the calculation was performed for the annihilation of positrons from the delocalized state (bulk), and for the annihilation of positrons trapped by $V_{\mathrm{Ga}}, V_{\mathrm{N}}$, divacancy $\left(V_{\mathrm{Ga}} V_{\mathrm{N}}\right)$, and quadravacancy $\left[\left(V_{\mathrm{Ga}} V_{\mathrm{N}}\right)_{2}\right]$. Since the annihilation of positrons trapped by $V_{\mathrm{N}}$ has only a small effect on the $(S, W)$ value, $V_{\mathrm{N}}$ is not the detectable vacancy-type defects. Note that the increase in the open volume of the defects results in a rightward shift in the $S$ - $W$ plot.

The calculated $(S, W)$ value for bulk GaN locates to the lower right of the value for HVPE-GaN. This difference might be due to several causes such as the limitations of first-principles calculations applied to Doppler broadening spectra, ${ }^{21}$ or the experimental background. For undoped and Er-doped $\mathrm{GaN}$ with $[\mathrm{Er}] \leqslant 1.5$ at. $\%$, the $(S, W)$ values lie on the line connecting the values of bulk and $V_{\mathrm{Ga}}$. This suggests that the major species of defects detected by positrons was $V_{\mathrm{Ga}}$, and their concentration increased with increasing $\mathrm{Er}$ concentration. According to the trapping model of positrons, the observed $S$ value is a weighted average between the characteristics $S$ values corresponding to the annihilation of positrons in bulk $\left(S_{b}\right)$ and in defects $\left(S_{d}\right): S=S_{b}\left(1-F_{d}\right)+S_{d} F_{d}$, where $F_{d}$ is the trapping fraction of positrons by the defects. $F_{d}$ is given by the relation $F_{d}=\mu_{d} C_{d} /\left(\lambda_{b}+\mu_{d} C_{d}\right)$, where $\mu_{d}$ is the positron trapping coefficient $\left(3 \times 10^{15} \mathrm{~s}^{-1}\right),{ }^{14} \lambda_{b}$ is the annihilation rate of positrons from the delocalized state $\left(6.54 \times 10^{9} \mathrm{~s}^{-1}\right),{ }^{28}$ and $C_{d}$ is the defect concentration. The calculated characteristic $S$ value for $V_{\mathrm{Ga}}$ was given by $1.069 S_{b}$. Using the experimentally determined bulk $S$ value, the defect concentrations were calculated and the results are shown in the inset of Fig. 2. From Figs. 2 and 5, we can conclude the following. The $V_{\mathrm{Ga}}$ concentration for the undoped $\mathrm{GaN}$ film was $5 \times 10^{16} \mathrm{~cm}^{-3}$, and it increased with increasing Er concentration at $[\mathrm{Er}] \leqslant 1.5$ at. $\%$. The $V_{\mathrm{Ga}}$ concentration reached $3 \times 10^{18} \mathrm{~cm}^{-3}$ at $[\mathrm{Er}]=1.5$ at. $\%$, and the introduction of larger vacancy-type defects such as $V_{\mathrm{Ga}} V_{\mathrm{N}}$ started at $[\mathrm{Er}]=3.3$ at. $\%$.

The estimated energy level of the defect complex be- tween Er and $V_{\mathrm{N}}\left(\mathrm{Er}_{\mathrm{Ga}}-V_{\mathrm{N}}\right)$ was about $0.2 \mathrm{eV}$ below the conduction band, ${ }^{6}$ and the IR emission was associated with the energy transfer from the electrons bound to $\mathrm{Er}_{\mathrm{Ga}}-V_{\mathrm{N}}$ to the $4 f$ core state of Er. ${ }^{5,29}$ The behavior of the $(S, W)$ values (Fig. 5) is well explained by the introduction of $V_{\mathrm{Ga}}$ and additional vacancy clusters. $V_{\mathrm{Ga}}$ has a deep triple acceptor level within about $1 \mathrm{eV}$ of the valence-band maximum. ${ }^{30}$ Thus, it would not be the defect participating in the Er-related fluorescence through the energy transfer. ${ }^{6}$ Walukiewicz et al. ${ }^{30}$ suggested that the formation energy of native defects is dependent on the Fermi level $\left(E_{\mathrm{F}}\right)$ position with respect to the Fermi stabilization energy $\left(E_{\mathrm{FS}}\right)$, which is the average energy level of native defects. In this model (the amphoteric defect model), acceptor-(donor)like defects are predominantly formed for $E_{\mathrm{F}}>E_{\mathrm{FS}}\left(E_{\mathrm{F}}<E_{\mathrm{FS}}\right)$, and the condition $E_{\mathrm{F}}=E_{\mathrm{FS}}$ is achieved when the donor- and acceptor-like defects are incorporated at rates where they compensate each other. Thus, both acceptor and donor-type defects are introduced by Er doping, and $V_{\mathrm{N}}$ enhances the intraemission of Er. The emission properties of $\mathrm{RE}$ atoms are strongly affected by the local symmetry of the host. ${ }^{2}$ Although Er- $V_{\mathrm{Ga}}$ may not participate to the energy transfer directly, they could change the chemical bonding structure of the ligands nearest to RE atoms, and affect the emission. The present experiments suggests that defect engineering to control the defect species and their concentrations while preserving a highly crystalline GaN matrix is one key to increasing the emission rate of the intra- $4 f$ shell transitions from an RE metal.

\section{SUMMARY}

We used a monoenergetic positron beam to study vacancy-type defects in Er-doped GaN grown by MBE. Doppler broadening spectra of the annihilation radiation were measured as a function of the incident energy of positrons for samples with an Er concentration between 0.3 and 6.0 at. \%. Doppler broadening spectra were theoretically calculated from valence-electron wavefunctions obtained by the PAW method to identify defect species in GaN. We have obtained a clear correlation between the point defects and the intra- $4 f$ transitions of Er. For the undoped and Er-doped GaN with $[\mathrm{Er}]=0.3-1.5$ at. $\%$, the major defect species was identified as a $\mathrm{Ga}$ vacancy $\left(V_{\mathrm{Ga}}\right)$ and its concentration increased with increasing Er concentration; the $V_{\mathrm{Ga}}$ concentration reached $3 \times 10^{18} \mathrm{~cm}^{-3}$ at $[\mathrm{Er}]=1.5$ at. $\%$. For the sample with $[\mathrm{Er}]$ $=3.3$ at. $\%$, the maximum integrated intensity of PL was observed, while the introduction of additional vacancies such as divacancies started at this doping concentration. The Er depth distribution agreed well with that of vacancy-type defects, and the Er accumulation near the GaN/substrate interface introduced vacancies in the same region. The major vacancy-type defects detected by positrons do not directly participate in the Er-related fluorescence, but $\mathrm{N}$ vacancies $\left(V_{\mathrm{N}}\right)$ might enhance the intraemission, where both the concentrations of $V_{\mathrm{N}}$ and $V_{\mathrm{Ga}}$ were expected to increase at the same time according to the amphoteric defect model. 


\section{ACKNOWLEDGMENTS}

Part of this study was financially supported by the Budget of the Ministry of Education, Culture, Sports, Science, and Technology for "Optoelectronics Frontier by Nitride Semiconductor-Ultimate Utilization of Nitride Semiconductor Material Potential" and for "Nuclear Research" based on screening and counseling by the Atomic Energy Commission, and by the Next Generation Super Computing Project, Nanoscience Program, MEXT, Japan. One of authors (A.U.) thanks Mitsubishi Chemical Corporation for supplying HVPE-GaN

${ }^{1}$ Rare Earth Doped Semiconductors III, in Proceedings of E-MRS Symposium Spring 2000, edited by J. M. Zavada, T. Gregorkiewicz, and A. Steckl; Mater. Sci. Eng., B 81, (2001).

${ }^{2}$ R. G. Wilson, R. N. Schwartz, C. R. Abernathy, S. J. Pearton, N. Newman, M. Rubin, T. Fu, and J. M. Zavada, Appl. Phys. Lett. 65, 992 (1994).

${ }^{3}$ A. J. Steckl and R. Birkhahn, Appl. Phys. Lett. 73, 1700 (1998).

${ }^{4}$ U. Wahl, E. Alves, K. Lorenz, J. G. Correia, T. Monteiro, B. De Vries, A Vantomme, and R. Vianden, Mater. Sci. Eng., B 105, 132 (2003).

${ }^{5}$ C. Ugolini, N. Nepal, J. Y. Lin, H. X. Jiang, and J. M. Zavada, Appl. Phys. Lett. 90, 051110 (2007).

${ }^{6}$ J.-S. Filhol, R. Jones, M. J. Shaw, and P. R. Briddon, Appl. Phys. Lett. 84, 2841 (2004).

${ }^{7}$ E. E. Nyein, U. Hömmerich, J. Heikenfeld, D. S. Lee, A. J. Steckl, and J. M. Zavada, Appl. Phys. Lett. 82, 1655 (2003).

${ }^{8}$ A. Ishizumi, J. Sawahata, K. Akimoto, and Y. Kanemitsu, Appl. Phys. Lett. 89, 191908 (2006).

${ }^{9}$ R. Krause-Rehberg and H. S. Leipner, Positron Annihilation in Semiconductors, Solid-State Sciences 127 (Springer, Berlin, 1999).

${ }^{10}$ K. Saarinen, T. Suski, I. Grzegory, and D. C. Look, Phys. Rev. B 64 233201 (2001).
${ }^{11}$ S. F. Chichibu, A. Setoguchi, A. Uedono, K. Yoshimura, and M. Sumiya, Appl. Phys. Lett. 78, 28 (2001).

${ }^{12}$ J. Moxom, J. Xu, R. Suzuki, T. Ohdaira, G. Brandes, and J. S. Flynn, J. Appl. Phys. 92, 1898 (2002).

${ }^{13}$ A. Uedono, H. Bang, K. Horibe, S. Morishima, and K. Akimoto, J. Appl. Phys. 93, 5181 (2003).

${ }^{14}$ J. Oila, J. Kivioja, V. Ranki, K. Saarinen, D. C. Look, R. J. Molnar, S. S. Park, S. K. Lee, and J. Y. Han, Appl. Phys. Lett. 82, 3433 (2003).

${ }^{15}$ S. Zeng, G. N. Aliev, D. Wolverson, J. J. Davies, S. J. Bingham, D. A. Abdulmalik, P. G. Coleman, T. Wang, and P. J. Parbrook, Appl. Phys. Lett. 89, 022107 (2006)

${ }^{16}$ S. Hautakangas, I. Makkonen, V. Ranki, M. J. Puska, K. Saarinen, X. Xu, and D. C. Look, Phys. Rev. B 73, 193301 (2006).

${ }^{17}$ S. Morishima, T. Maruyama, K. Akimoto, J. Cryst. Growth 209, 378 (2000).

${ }^{18}$ A. van Veen, H. Schut, J. de Vries, R. A. Hakvoort, and M. R. Ijpma, AIP Conf. Proc. 218, 171 (1990).

${ }^{19}$ P. E. Blöchl, Phys. Rev. B 50, 17953 (1994).

${ }^{20}$ S. Ishibashi, T. Tamura, S. Tanaka, M. Kohyama, and K. Terakura (unpublished).

${ }^{21}$ S. Ishibashi, Mater. Sci. Forum 445-446, 401 (2004).

${ }^{22}$ E. Boroński and R. M. Nieminen, Phys. Rev. B 34, 3820 (1986)

${ }^{23}$ J. P. Perdew, K. Burke, and M. Ernzerhof, Phys. Rev. Lett. 77, 3865 (1996).

${ }^{24}$ A. Uedono, S. F. Chichibu, Z. Q. Chen, M. Sumiya, R. Suzuki, T. Ohdaira, T. Mikado, T. Mukai, and S. Nakamura, J. Appl. Phys. 90, 181 (2001).

${ }^{25}$ M. Thaik, U. Hömmerich, R. N. Schwartz, R. G. Wilson, and J. M. Zavada, Appl. Phys. Lett. 71, 2641 (1997).

${ }^{26}$ T. Monteiro, J. Soares, M. R. Correia, and E. Alves, J. Appl. Phys. 89, $6183(2001)$

${ }^{27}$ C. G. Van de Walle and J. Neugebauer, J. Appl. Phys. 95, 3851 (2004).

${ }^{28}$ A. Uedono, K. Ito, H. Nakamori, K. Mori, Y. Nakano, T. Kachi, S. Ishibashi, T. Ohdaira, and R. Suzuki, J. Appl. Phys. 102, 084505 (2007).

${ }^{29}$ S. F. Song, W. D. Chen, C. Zhang, L. Bian, C. C. Hsu, L. W. Lu, Y. H. Zhang, and J. Zhu, Appl. Phys. Lett. 86, 152111 (2005).

${ }^{30}$ W. Walukiewicz, Physica B 302-303, 123 (2001). 